\section{Pawel Golda}

Université d'Opole

Pologne

Université de Silésie, Katowice

Pologne

Université Sorbonne Paris Nord

France

(D) https://orcid.org/0000-0001-5505-7731

Natalia Żywicka

Université Pédagogique de Cracovie

Pologne

(iD https://orcid.org/0000-0002-8832-5576

\section{Vanessa Ferreira Vieira}

Université de São Paulo

Brésil

(iD https://orcid.org/0000-0003-2483-570X

\section{S'attaquer à la suprématie du masculin sur le féminin : le français inclusif dans les publications des universités françaises dans les réseaux sociaux}

Combating the supremacy of the masculine over the feminine:

Inclusive French in social media publications of French universities

\begin{abstract}
This paper aims to examine the use of inclusive French in the Internet publications of Paris universities on their social media. Three higher education institutions were selected: Paris Dauphine-PSL University, Gustave Eiffel University, and Sorbonne Paris North University. The publications were obtained from Facebook, Instagram, and LinkedIn. Firstly, the groups of people to whom the use of inclusive French referred were considered. The second question was about the practices used to make the French language inclusive. Eight practices were observed and are described in the paper. Also, the frequency of gender-neutral language was a point of interest. The research corpus is available online: https://tiny.pl/9rcdj.
\end{abstract}

\title{
Keywords
}

Gender-neutral language, generic masculine, inclusive writing, inclusive French, sociolinguistics 


\section{Introduction}

Dans la seconde moitié du $\mathrm{XX}^{\mathrm{e}}$ siècle, une série de mobilisations a placé au cœur d'un débat la nécessité de penser à des pratiques langagières axées sur la substitution du masculin considéré générique par des formes faisant ressortir le féminin dans la langue. Les mouvements féministes des années 1970 ont ébranlé les espaces politiques à travers les discours sur le sexisme linguistique (J. Abbou et al., 2018 : 137-138). En revanche, les honorables Claude Lévi-Strauss et Georges Dumézil, membres élus à l'Académie française dans la même décennie, sont restés hostiles aux changements des principes du français qui pourraient mettre la langue en désordre ${ }^{1}$.

Au cours des derniers mois de l'année 2017, les controverses sur l'usage du langage inclusif se sont intensifiées. Le 22 septembre, un article du journal Le Figaro a dévoilé l'utilisation de l'écriture inclusive dans un manuel scolaire destiné aux élèves du primaire ${ }^{2}$. En octobre 2017, l'Académie française a adopté à l'unanimité de ses membres une déclaration sur l'écriture inclusive en la considérant une « aberration » et un " péril mortel » à cause de la multiplication des marques orthographiques susceptibles de rendre la langue française désunie et illisible ${ }^{3}$. En novembre 2017, le site Slate.fr a fait paraître un manifeste signé par 314 membres du corps professoral de tous les niveaux déclarant ne plus enseigner la formule «Le masculin l'emporte sur le féminin » ${ }^{4}$. Le 28 février 2019, l'Académie française a adopté un rapport sur la féminisation des noms de métiers et de fonctions ${ }^{5}$. Deux ans plus tard, en mai 2021, est parue une lettre ouverte de l'Académie française dans laquelle les Immortels admettent que l'écriture inclusive s'inscrit dans la lutte contre les discriminations fondées sur le sexe, mais qu'elle est « nuisible à la pratique et à l'intelligibilité de la langue française $»^{6}$. Les Académiciens sou-

${ }^{1}$ Académie française (2014). La féminisation des noms de métiers, fonctions, grades ou titres. Mise au point de l'Académie française. http://www.academie-francaise.fr/sites/academie-francaise. fr/files/feminisation_2014.pdf (consulté le 15.03.2021).

${ }^{2}$ https://www.lefigaro.fr/actualite-france/2017/09/22/01016-20170922ARTFIG00300-unmanuel-scolaire-ecrit-a-la-sauce-feministe.php (consulté le 14.03.2021).

${ }^{3}$ Académie française (2017). Déclaration de l'Académie française sur l'écriture dite "inclusive » adoptée à l'unanimité de ses membres dans la séance du jeudi 26 octobre 2017. http://www. academiefrancaise.fr/actualites/declaration-de-lacademie-francaise-sur-lecriture-dite-inclusive (consulté le 14.03.2021).

${ }^{4} \mathrm{http} / / / \mathrm{www}$. slate.fr/story/153492/manifeste-professeurs-professeures-enseignerons-plus-masculin-emporte-sur-le-feminin (consulté le 14.03.2021).

5 Académie française (2019). La féminisation des noms de métiers et de fonctions. https://www. academie-francaise.fr/sites/academie-francaise.fr/files/rapport_feminisation_noms_de_metier_et_ de_fonction.pdf (consulté le 14.03.2021).

${ }^{6}$ Académie française (2021). Lettre ouverte sur l'écriture inclusive. https://www.academiefrancaise.fr/actualites/lettre-ouverte-sur-lecriture-inclusive (consulté le 12.07.2021). 
lignent qu'en préconisant une réforme immédiate et globale de l'orthographe, les défenseurs de l'écriture inclusive perturbent le rythme du développement de la langue. Les membres de l'Académie française constatent que l'écriture épicène est un obstacle pour les personnes en situation de handicap cognitif, notamment la dyslexie, la dysphasie ou l'apraxie, ce qui « a pour effet concret d'aggraver des inégalités $»$.

Cet article propose une analyse du langage inclusif utilisé par trois universités parisiennes dans leurs réseaux sociaux, visant à comprendre quelles sont les pratiques du français inclusif (désormais FI) appliquées dans leurs publications Internet. Cette étude ne se limite pas à réfléchir sur les stratégies possibles pour surpasser les règles qui voient le masculin prévaloir sur le féminin, mais également sur les pratiques utilisées pour désigner un groupe de personnes.

\section{Prolégomènes théoriques}

\subsection{Approche définitoire du FI}

Selon Maria Candea (J. Abbou et al., 2018 : 133), l'écriture inclusive est un large éventail de pratiques qui sont utilisées pour créer un langage sans discrimination à l'égard du genre féminin, mais aussi des personnes qui s'identifient comme non-binaires. Julie Abbou (2018: 133) précise que ce phénomène peut se réaliser de diverses façons telles que :

- l'emploi de formules syntaxiques impersonnelles, p. ex. penser au lectorat ;

- les choix lexicaux, p. ex. droits humains à la place de droits de l'homme;

- les jeux de flexion, p. ex. auteure, auteur ;

- les méthodes typographiques, p. ex. certaine.

Danielle Omer (2020 : 187) évoque que depuis 2012, les écoles françaises veulent être « inclusives " afin d'offrir des conditions adéquates à tous les élèves, notamment à ceux qui sont en situation de handicap. La chercheuse précise que l'écriture inclusive consiste à s'éloigner du féminin et du masculin lorsque l'on fait référence aux individus ainsi qu'à mettre fin à l'utilisation du masculin générique quand le discours s'adresse aussi aux femmes.

Alpheratz (2019 : 53-54) évoque que l'écriture inclusive désigne des processus langagiers qui visent à inclure les non-représentés ainsi que l'éthique qui consiste à assurer « la visibilité/valorisation/prise en compte/reconnaissance » des groupes minorisés dans les discours. Toujours dans la même référence, on définit le FI comme une variété de la langue caractérisée par l'emploi des techniques qui

\footnotetext{
${ }^{7}$ Ibidem.
} 
permettent d'éviter la propagation des hiérarchies symboliques et sociales basées sur la discrimination en termes de sexe, genre, âge, orientation sexuelle ou classe socioprofessionnelle. Cette définition présente le FI dans un sens beaucoup plus large que les définitions proposées par J. Abbou (2018 : 133) et par D. Omer (2020 : 187). Notons que dans notre recherche nous ne prenons en considération que la discrimination fondée sur le sexe et le genre.

\subsection{Dichotomie des genres grammaticaux}

Le genre en français est de nature dichotomique (D. Elmiger, $2018: 1$; C. Fairon, A.-C. Simon, 2018 : 88). La corrélation qui existe entre deux genres du français - le masculin et le féminin - est fondée sur la hiérarchie avec la domination masculine (L. Bereni et al., 2012 : 9). Remarquons que cette domination est solidement implantée dans la langue française. Le genre masculin peut englober le genre féminin et le rendre invisible puisque l'accord correspond toujours au masculin pluriel dans le cas d'un groupe mixte. En outre, le genre masculin à valeur générique permet d'identifier les personnes indépendamment de leur sexe (L. Michel, $2016: 3$ ).

Dans la déclaration de l'Académie française La féminisation des noms de métiers, fonctions, grades ou titres, il est constaté que deux genres grammaticaux constituent une sorte de limitation et, par conséquent, il a fallu en choisir un qui puisse être utilisé de manière générique ${ }^{8}$. Flora Bolter $(2019: 2)$ rappelle dans la même veine que le genre neutre latin était couvert en français par le genre masculin. Néanmoins, la valeur générique du masculin peut s'interpréter de différentes façons, ce qui peut entraîner certains problèmes. Nous pouvons l'utiliser par rapport à un individu de genre masculin, à un groupe neutre, ou à un individu ou un groupe sans distinction de genre (P. Gygax, U. Gabriel, S. Zufferey, 2019 : 61).

Daniel Elmiger (2020 : 697) montre les premières occurrences du féminin générique. Selon l'auteur, dans l'espace francophone le féminin à valeur générique est présent dans des livres adressés aux enfants ${ }^{9}$ ainsi que sur les comptes Twitter de Maria Candea (@MarCandea) et de Martin Winckler (@MartinWinckler). D. Elmiger (2020 : 697) rappelle aussi qu'à l'Université de Neuchâtel le règlement des Sciences de l'éducation pour l'année universitaire 2000/2001 a indiqué que le féminin " étudiantes » devait être utilisé en vue " de simplification et d'entraînement ». De surcroît, le féminin générique est imposé dans les statuts de cet établissement depuis 2018.

Le classement par genre impose l'identification, parfois accablante, avec l'une des deux catégories. Il convient de mettre en évidence la présence des personnes

\footnotetext{
${ }^{8}$ Académie française (2014). La féminisation des noms de métiers, fonctions, grades ou titres. Mise au point de l'Académie française.

${ }^{9}$ https://www.delta-atled.org (consulté le 23.02.2021).
} 
qui ne sont pas incluses dans cette division. Il s'agit notamment des personnes non-binaires (J. Abbou et al., 2018 : 133) intersexuelles et transsexuelles (D. Elmiger, 2018 : 909). Rappelons que le sexe est fondé sur des aspects physiques, et que le genre se base sur ce qu'une personne « performe, du point de vue social». Il s'agit des rôles et des comportements liés à l'appartenance au genre féminin ou masculin, ou qui sortent de cette classification (D. Elmiger, 2019 : 617).

\subsection{Pratiques du FI}

\subsubsection{Double flexion totale et partielle}

L'absence d'égalité entre les formes féminines et masculines dans le discours peut être compensée par leur utilisation simultanée. Alpheratz (2019 : 60-61) distingue la double flexion totale et la double flexion partielle. La première pratique s'appuie sur le remplacement de la forme masculine par le dédoublement des formes. Chez D. Elmiger (2017 : 42), ce processus est nommé le doublet intégral ou le doublet entier. Les exemples de ce procédé peuvent être les Suissesses et les Suisses ou les nouveaux étudiants et les nouvelles étudiantes. Nous pouvons trouver des doublets intégraux dans des situations d'homophonie, p. ex. les martyres et les martyrs. Notons que cette technique peut entraîner des problèmes tels que :

- la nécessité du choix de l'ordre dans lequel les formes sont présentées (féminin/ masculin ou masculin/féminin) (D. Elmiger, $2014: 156$ ) ;

- le manque de l'économie linguistique (D. Elmiger, 2017 : 42) ;

- la difficulté de l'accord (l'accord de proximité, « au genre le plus noble » ou l'accord de majorité) (É. Viennot, 2020).

Quant à la double flexion partielle, elle inclut le genre masculin et le genre féminin, mais ce dernier est indiqué par l'addition de la marque morphologique (Alpheratz, 2019 : 61). D. Elmiger (2017 : 42) nomme cette technique les doublets abrégés. Elle dispense du dédoublement des formes identiques, mais les terminaisons visibles portant les désignations de genre persistent. Notons que cette pratique se caractérise par un grand nombre de signes utilisés :

- les tirets : regroupé-e, divers-es, masqué-e-s ;

- les majuscules : manifestantE, mauvaisES ;

- le souligné : sauvages (J. Abbou, 2011 : 61-63);

- les parenthèses : les étudiant(e)s ;

- les barres obliques : les étudiant/e/s;

- les points médians : les étudiante:s;

- les points : les étudiant.e.s ;

- les tirets bas : l'étudiant_e (D. Elmiger, 2017 : 43) ;

- les virgules : des fanfaron,ne,s; 
- d'autres symboles : dérivé€s, pauvre $\sim$ sse $\sim S$, Tou[te]s les product $<$ eur[s]|rice[s], tou:t;e,s, eלuxslles ;

- la différence de couleur : les éternelles étudiantes, les consommateurices (R. J. Aeschlimann, 2017 : 531-560).

Éliane Viennot (2020) constate que certains de ces signes sont idéologiquement ou techniquement imparfaits, ou bien ils sont imparfaits dans ces deux critères. Quant aux parenthèses, la chercheuse remarque qu'elles indiquent la valeur subordonnée. Le trait d'union rend le mot séparable et, par conséquent, il peut se diviser en deux lignes. De surcroît, il permet d'orthographier les mots composés. En ce qui concerne la barre oblique, elle fait appel à la variante « et/ ou ». Il y a également des problèmes liés au point. À savoir, il crée de faux liens hypertextes qui se mettent automatiquement en évidence. Il est également possible d'utiliser les majuscules, néanmoins, cette méthode accorde trop d'attention au genre féminin en lui donnant plus de valeur par rapport au genre masculin. S'agissant du point médian, il n'a pas d'autres fonctions, valeurs, ni significations, qu'elles soient positives ou négatives. Aussi semble-t-il le plus approprié.

\subsubsection{Formule englobante et nom collectif}

La formule englobante est une technique qui fait référence à un groupe mixte sans indiquer le genre des individus composant ce groupe. Elle permet non seulement de s'éloigner du masculin générique, mais aussi d'éviter la division binaire. Comme il s'agit d'une pratique qui est moins décortiquée dans les recherches linguistiques, nous allons nous servir d'exemples. Voici une citation tirée de l'un des articles d'Isabelle Thireau :

Les lettres collectives peuvent comporter des dizaines de signatures, ou porter le nom et les coordonnées des porte-parole du groupe concerné, ou encore conclure sur une formule englobante « les milliers de résidents du quartier de $\mathrm{X}$ de la ville $\mathrm{Y}$ ».

(2014: 114-115)

Nous voyons, grâce à cet exemple, que la formule englobante peut conclure une lettre collective. Elle peut remplacer des milliers ou des dizaines de signatures, ce qui met en évidence la valeur collective de ce procédé. D'autres exemples de cette méthode de l'inclusion sont :

- le corps professoral qui remplace les enseignants ou les professeurs ;

- le corps médical qui remplace les médecins;

- le secteur agricole qui remplace les agriculteurs;

- la population française qui remplace les Français.

Une autre technique du FI qui s'appuie sur le groupement est l'utilisation du nom collectif (J. Abbou et al., 2018 : 133), p. ex. l'emploi du mot public au lieu 
du nom spectateurs. Le nom collectif est un mot utilisé au singulier pour désigner des groupements d'objets ou d'êtres animés, p. ex. : bouquet, équipe, comité (M. Lammert, 2017 : 101). Les noms collectifs se décrivent généralement par la relation sémantique qui existe entre un tout et ses éléments, p. ex : armée/soldat, équipeljoueur, public/spectateur ${ }^{10}$.

\subsubsection{Formule non-genrée}

Maria Candea (J. Abbou et al., 2018 : 133) donne une signification plus vaste au nom collectif. La chercheuse appelle le nom collectif le cas où l'on privilégie l'utilisation de l'expression carte électorale au lieu de carte d'électeur. Il s'agit à ce point d'une pratique qui n'a pas été suffisamment abordée dans les recherches linguistiques pour l'instant. Nous constatons la nécessité d'un nouveau terme qui pourrait dénommer cette technique du FI et proposons la dénomination « formule non-genrée ». Cette pratique consiste à remplacer un nom suivi d'un syntagme prépositionnel composé de la préposition de et d'un nom de genre masculin par un nom suivi d'un adjectif. Nous considérons que l'expression Droits humains remplaçant Droits de l'Homme ${ }^{11}$ est un bon exemple de la formule non-genrée.

\subsubsection{Forme épicène et abréviation}

La pratique suivante du FI est l'emploi des formes épicènes, c'est-à-dire des mots qui représentent la même forme pour le féminin et pour le masculin. Ils peuvent se présenter sous la forme des mots se terminant par la lettre - $e$ :

- les noms, p. ex. bibliothécaire, chimiste ;

- les adjectifs, p. ex. lisible, saumâtre;

- les noms et les adjectifs, p. ex. responsable, locataire (D. Elmiger, 2017 : 44).

Nous trouvons également des lexèmes qui ne se terminent pas avec $-e$, mais que nous considérons comme épicènes, p. ex. sympa, zen (E. Dawes, 2003 : 196).

Il sied de mentionner que les formes abrégées des noms ont la même caractéristique. Le TLFi présente la définition suivante de l'abréviation : "Procédé par lequel on obtient une représentation graphique tronquée, mais suffisamment claire, d'un signe plus long $\rangle^{12}$. L'abréviation vise à garder le début d'une chaîne phonétique ou graphique. L'effondrement des terminaisons que supposent les procédés d'abréviation conduit souvent à des formes épicènes qui ne sont pas expo-

${ }^{10}$ Exemples de M. Lammert, M. Lecolle, 2014.

${ }^{11}$ https://www.assemblee-nationale.fr/dyn/15/rapports/ega/115b1095_rapport-information (consulté le 13.03.2021).

${ }^{12}$ https://www.cnrtl.fr/definition/abr\%C3\%A9viations (consulté le 16.07.2021). 
sées à l'alternance. Ils sont invariables en genre, p. ex. le/la prof, l'écolo, le/la pdg (D. Elmiger, 2008 : 120). Nous distinguons trois types d'abréviations :

- le sigle qui contient des lettres initiales de mots, p. ex. le/la sdf (sans domicile fixe $^{13}$;

- l'acronyme qui est un sigle qui se prononce comme un mot ordinaire, p. ex. lel la PRAG (professeur(e) agrégé(e)) ${ }^{14}$;

- la troncation qui implique la suppression de syllabes, p. ex. le/la psy $(\text { psychologue })^{15}$.

\subsubsection{Féminisation}

Dans le rapport La féminisation des noms de métiers et de fonctions du 28 février 2019, l'Académie française constate :

En ce début de $\mathrm{XXI}^{\mathrm{e}}$ siècle, tous les pays du monde, et en particulier la France et les autres pays entièrement ou en partie de langue française, connaissent une évolution rapide et générale de la place qu'occupent les femmes dans la société, de la carrière professionnelle qui s'ouvre à elles, des métiers et des fonctions auxquels elles accèdent sans que l'appellation correspondant à leur activité et à leur rôle réponde pleinement à cette situation nouvelle. Il en résulte une attente de la part d'un nombre croissant de femmes, qui souhaitent voir nommer au féminin la profession ou la charge qu'elles exercent, et qui aspirent à voir combler ce qu'elles ressentent comme une lacune de la langue ${ }^{16}$.

Dans le même document, on admet qu'il n'existe aucun obstacle de principe à la féminisation des noms de métiers et de professions, et que le français possède des règles morphologiques dont il n'est pas possible de s'affranchir quitte à bouleverser le système de la langue. Il y a également la possibilité d'ajouter le mot femme au nom de genre masculin qui reste dans ce cas inchangé, p. ex. femme écrivain, femme peintre, pourtant certains linguistes recommandent d'en éviter (C. Fairon, A.-C. Simon, 2018 : 516).

Il faut souligner que les formes masculines sont plus attendues pour désigner les femmes exerçant les hautes fonctions publiques, p. ex. président, ministre, académicien (A. Chatard et al., 2005 : 252). Dans la déclaration de l'Académie française de 2014, on met en évidence que l'utilisation du masculin à valeur générique ou «non marqué » est une bonne solution lorsque le sexe de l'individu n'est pas plus important que d'autres caractéristiques individuelles. L'Académie consi-

\footnotetext{
${ }^{13}$ https://www.cnrtl.fr/definition/sigle (consulté le 10.04.2021).

${ }^{14} \mathrm{https} / / / \mathrm{www}$.larousse.fr/dictionnaires/francais/acronyme/858 (consulté le 10.04.2021).

${ }^{15} \mathrm{https} / / / \mathrm{www} . c n r t l . f r / d e f i n i t i o n /$ troncation (consulté le 10.04.2021).

${ }^{16}$ Académie française (2019). La féminisation des noms de métiers et de fonctions.
} 
dère un barbarisme les féminisations une fois qu'elles sont contraires aux règles de dérivation ${ }^{17}$.

\section{Présentation du corpus}

Le but principal de notre article est de voir si et comment les pratiques du FI sont utilisées dans les publications des universités françaises dans leurs réseaux sociaux officiels. Nous avons choisi trois établissements d'enseignement supérieur français, notamment l'Université Paris Dauphine-PSL, l'Université Gustave Eiffel et l'Université Sorbonne Paris Nord. Toutes les trois institutions sont placées dans la région parisienne. Précisons qu'il s'agit des pages principales de ces trois établissements, et que nous n'avons pas pris en compte les contenus ajoutés sur les pages de leurs fractions (p. ex. facultés, laboratoires, écoles doctorales). Qui plus est, nous avons choisi trois médias sociaux : Facebook, Instagram et LinkedIn. L'analyse a pris en compte les textes publiés du 01.12.2020 au 31.01.2021.

Il sied de stipuler que les éléments textuels publiés en tant que stories $^{18}$ n'ont pas constitué l'objet de l'observation. S'agissant des partages sur Facebook et sur LinkedIn, nous avons pris en considération uniquement les textes ajoutés par les universités aux éléments partagés.

L'analyse des textes a été effectuée à travers une observation attentive. Nous avons rendu notre corpus disponible sur Dropbox sous forme d'un fichier PDF. Il est accessible sur le lien suivant ${ }^{19}$ :

https://www.dropbox.com/s/9otvj0yz0sc4vzj/S\%E2\%80\%99attaquer\%20 $\%$ C3\%A0\%201a\%20supr\%C3\%A9matie $\% 20$ du $\% 20$ masculin $\% 20$ sur $\% 201$ e $\% 20$ f\%C3\%A 9minin $\% 20-\% 201$ e $\% 20$ fran $\%$ C3\%A7ais $\% 20$ inclusif $\% 20$ dans $\% 201$ es $\% 20$ publications $\% 20 \mathrm{des} \% 20$ universit $\% \mathrm{C} 3 \%$ A 9 s\%20fran $\% \mathrm{C} 3 \%$ A7aises $\% 20$ dans $\% 20$ les $\% 20$ r\%C3\%A9seaux\%20sociaux\%20-\%20CORPUS\%20DE\%20LA\%20RECHERCHE.pdf?dl=0

${ }^{17}$ Académie française (2014). La féminisation des noms de métiers, fonctions, grades ou titres. Mise au point de l'Académie française.

${ }^{18}$ Les stories sont les publications, à savoir photos ou vidéos, éphémères. Les éléments y partagés sont disponibles pendant 24 heures. Les stories ne sont pas visibles dans le profil ou dans le fil d'actualité. Cette fonction est disponible sur tous les trois réseaux sociaux choisis pour l'étude.

${ }^{19}$ Il existe également une version raccourcie du lien : https://tiny.pl/9rcdj. 


\section{Partie analytique}

\subsection{Observations préliminaires}

Pendant la période de l'analyse, 356 contenus ont été publiés et sont devenus l'objet de notre recherche. Dans ce total, 154 publications sont apparues en décembre 2020 et 202 éléments sont apparus en janvier 2021. Tous les trois établissements d'enseignement supérieur ont augmenté leur activité dans les réseaux sociaux en janvier 2021 par rapport au décembre 2020. Dans le tableau 1, nous présentons tous les nombres de publications prises en considération dans la recherche.

Tableau 1

Nombre exact de publications

\begin{tabular}{|l|c|c|c|c|}
\hline \multicolumn{1}{|c|}{ Établissement } & Facebook & Instagram & LinkedIn & TOTAL \\
\hline Université Paris Dauphine-PSL & 20 & 7 & 49 & 76 \\
\hline Université Gustave Eiffel & 58 & 2 & 54 & 114 \\
\hline Université Sorbonne Paris Nord & 100 & 16 & 50 & 166 \\
\hline TOTAL & 178 & 25 & 153 & 356 \\
\hline
\end{tabular}

La première étape de l'analyse a consisté à voir combien de notes publiées contenaient le FI. Nous avons constaté sa présence dans 156 de tous les 356 éléments analysés. Le tableau 2 présente les nombres de publications avec au moins une utilisation du FI.

Tableau 2

Nombre de publications contenant au moins une utilisation du FI

\begin{tabular}{|l|c|c|c|c|}
\hline \multicolumn{1}{|c|}{ Établissement } & Facebook & Instagram & LinkedIn & TOTAL \\
\hline Université Paris Dauphine-PSL & 12 & 4 & 27 & 43 \\
\hline Université Gustave Eiffel & 24 & 2 & 16 & 42 \\
\hline Université Sorbonne Paris Nord & 38 & 10 & 23 & 71 \\
\hline TOTAL & 74 & 16 & 66 & 156 \\
\hline
\end{tabular}

Étant donné qu'un contenu ajouté a pu comprendre plus d'un seul emploi du FI, le nombre d'utilisations concrètes de telles pratiques s'élève à un nombre plus important. Ainsi, nous avons sélectionné 279 utilisations du FI. Le tableau 3 rassemble toutes les informations sur les nombres d'emplois de telles techniques. 
Toutes les utilisations du FI

\begin{tabular}{|l|c|c|c|c|}
\hline \multicolumn{1}{|c|}{ Établissement } & Facebook & Instagram & LinkedIn & TOTAL \\
\hline Université Paris Dauphine-PSL & 18 & 5 & 48 & 71 \\
\hline Université Gustave Eiffel & 41 & 4 & 26 & 71 \\
\hline Université Sorbonne Paris Nord & 73 & 16 & 48 & 137 \\
\hline TOTAL & 132 & 25 & 122 & 279 \\
\hline
\end{tabular}

\subsection{Personnes à qui se réfère le FI}

L'une des questions que nous nous sommes posées lors de l'observation des publications était de savoir à qui se référaient les pratiques du FI. Nous avons effectué une division des destinataires du FI en sept groupes :

- les chercheurs,

- les doctorants,

- les étudiants,

- les doctorants/les étudiants (ensemble),

- les candidats,

- tout le monde,

- les autres (p. ex. journalistes, professionnels, activistes).

Le plus souvent le FI s'est référé aux chercheurs (127 cas). Ensuite, 92 utilisations ont été classées comme renvoyant au groupe " les autres ». Le tableau 4 présente au plus juste les données chiffrées concernant les destinataires du FI.

Personnes à qui s'est référé le FI

Tableau 4

\begin{tabular}{|l|c|c|c|c|}
\hline $\begin{array}{c}\text { Groupe de personnes } \\
\text { à qui s'est référé le FI }\end{array}$ & $\begin{array}{c}\text { Université } \\
\text { Paris } \\
\text { Dauphine-PSL }\end{array}$ & $\begin{array}{c}\text { Université } \\
\text { Gustave Eiffel }\end{array}$ & $\begin{array}{c}\text { Université } \\
\text { Sorbonne } \\
\text { Paris Nord }\end{array}$ & TOTAL \\
\hline Chercheurs & 27 & 17 & 83 & 127 \\
\hline Doctorants & 1 & 2 & 0 & 3 \\
\hline Étudiants & 10 & 17 & 16 & 43 \\
\hline Doctorants/Étudiants & 1 & 0 & 1 & 2 \\
\hline Candidats & 3 & 0 & 0 & 3 \\
\hline Tout le monde & 3 & 3 & 3 & 9 \\
\hline Autres & 26 & 32 & 34 & 92 \\
\hline
\end{tabular}




\subsection{Pratiques du FI présentes dans le corpus}

L'analyse de l'activité des universités dans les réseaux sociaux nous a permis d'observer huit procédés visant à rendre la langue française inclusive, notamment :

- la double flexion totale,

- la double flexion partielle,

- la formule englobante,

- le nom collectif,

- la formule non-genrée,

- la forme épicène,

- l'abréviation,

- la féminisation.

Ces techniques ont d'ores et déjà été définies dans la partie théorique de l'article. Toutes les informations concernant les nombres d'utilisations de ces méthodes sont rassemblées dans le tableau 5.

Tableau 5

Nombres d'utilisations des pratiques du FI

\begin{tabular}{|l|c|c|c|c|}
\hline \multicolumn{1}{|c|}{ Pratique } & $\begin{array}{c}\text { Université } \\
\text { Paris } \\
\text { Dauphine-PSL }\end{array}$ & $\begin{array}{c}\text { Université } \\
\text { Gustave Eiffel }\end{array}$ & $\begin{array}{c}\text { Université } \\
\text { Sorbonne } \\
\text { Paris Nord }\end{array}$ & TOTAL \\
\hline Double flexion totale & 3 & 6 & 12 & 21 \\
\hline Double flexion partielle & 4 & 17 & 0 & 21 \\
\hline Nom collectif & 7 & 15 & 14 & 36 \\
\hline Formule englobante & 10 & 4 & 7 & 21 \\
\hline Formule non-genrée & 9 & 9 & 15 & 33 \\
\hline Forme épicène & 25 & 9 & 34 & 68 \\
\hline Abréviation & 0 & 0 & 26 & 26 \\
\hline Féminisation & 13 & 11 & 29 & 53 \\
\hline
\end{tabular}

\subsection{Double flexion totale}

La double flexion totale a été observée 21 fois dans les 356 publications entrant dans le corpus de la recherche (tableau 6). L'exemplification démontre que de tels dédoublements peuvent jouer de différents rôles au sein de la phrase. Nous disposons d'exemples où la double flexion totale est présente dans : 
- le sujet de la phrase, p. ex. « Les femmes et les hommes ${ }^{20}$ de l'Université Gustave Eiffel vous souhaitent une bonne année 2021 ! » (UGE, $\mathrm{LI}^{21}$ ) ;

- le complément d'objet indirect, p. ex. «Bonne fin de journée à toutes et tous ! » (USPN, IG) ;

- l'apostrophe, p. ex. " Étudiantes, étudiants, contribuez à faire changer le regard sur le handicap » (USPN, LI).

Parmi les 21 exemples de la double flexion totale, nous avons trouvé 19 dédoublements de composants nominaux, 1 dédoublement de composants pronominaux (Celles et ceux) et 1 dédoublement composé des adjectifs indéfinis et des pronoms (Toutes celles et tous ceux).

Dans tous les exemples retrouvés, le premier élément de la double flexion totale était de genre féminin et le second était masculin. Tous les dédoublements de ce type étaient composés d'éléments au pluriel. Nous avons été en mesure de reconnaître trois types distincts de la jonction entre les mots dédoublés :

- la conjonction de coordination et : élément1 et élément2 (13 ex.);

— la virgule : élément1, élément2 (7 ex.) ;

— l'esperluette : élément1 \& élément2 (1 ex.).

Double flexion totale

Tableau 6

\begin{tabular}{|l|c|l|}
\hline $\begin{array}{c}\text { Double flexion } \\
\text { totale }\end{array}$ & $\begin{array}{c}\text { Nombre } \\
\text { d'emplois }\end{array}$ & \multicolumn{1}{|c|}{ Citation } \\
\hline celles et ceux & 1 & $\begin{array}{l}\text { « Bon courage pour les révisions à celles et ceux qui passent des } \\
\text { examens très prochainement ! » (USPN, IG) }\end{array}$ \\
\hline $\begin{array}{l}\text { chercheuses, } \\
\text { chercheurs }\end{array}$ & 6 & $\begin{array}{l}\text { «Chercheuses, chercheurs de l'Université Sorbonne Paris Nord, } \\
\text { de toutes disciplines [...] » (USPN, LI) }\end{array}$ \\
\hline $\begin{array}{l}\text { docteures et } \\
\text { docteurs }\end{array}$ & 1 & $\begin{array}{l}\text { « Jeunes docteures et docteurs en Sciences Humaines et Sociales } \\
{[\ldots] \text { (.. (UPD-PSL, LI) }}\end{array}$ \\
\hline $\begin{array}{l}\text { enseignantes } \\
\text { \& enseignants }\end{array}$ & 1 & $\begin{array}{l}\text { « Découvrez l'interview de trois enseignantes \& enseignants }- \\
\text { chercheurs des laboratoires et dans certains de nos masters ainsi } \\
\text { que d'un jeune entrepreneur alumnus ESPCI Paris - PSL [...] » } \\
\text { (UPD-PSL, LI) }\end{array}$ \\
\hline $\begin{array}{l}\text { étudiantes, } \\
\text { étudiants }\end{array}$ & 1 & $\begin{array}{l}\text { « Étudiantes, étudiants, contribuez à faire changer le regard sur } \\
\text { le handicap » (USPN, LI) }\end{array}$ \\
\hline
\end{tabular}

${ }^{20}$ Nous utilisons les caractères gras pour marquer les pratiques du FI dans les citations.

${ }^{21}$ Dans l'intégralité de l'article, toutes les publications sont référencées à l'aide des abrégés suivants : UPD-PSL pour l'Université Paris Dauphine-PSL, UGE pour l'Université Gustave Eiffel et $U S P N$ pour l'Université Sorbonne Paris Nord. En outre, nous introduisons des abrégés qui vont indiquer les réseaux sociaux : $F B$ pour Facebook, $I G$ pour Instagram, $L I$ pour LinkedIn. La référence «USPN, IG » signifie que le contenu cité a été publié sur Instagram de l'Université Sorbonne Paris Nord. Tous les liens permettant de retrouver ces publications dans l'environnement WEB se trouvent dans le corpus publié sur Dropbox : https://tiny.pl/9rcdj. 


\begin{tabular}{|l|c|l|}
\hline $\begin{array}{l}\text { les femmes et les } \\
\text { hommes }\end{array}$ & 3 & $\begin{array}{l}\text { « Les femmes et les hommes de l'Université Gustave Eiffel vous } \\
\text { souhaitent une bonne année 2021 ! » (UGE, IG) }\end{array}$ \\
\hline $\begin{array}{l}\text { toutes celles et } \\
\text { tous ceux }\end{array}$ & 1 & $\begin{array}{l}\text { " Je suis très honorée de recevoir cette distinction et je tiens à } \\
\text { exprimer toute ma reconnaissance à toutes celles et tous ceux qui } \\
\text { ont assuré la continuité d'activité de notre université » (USPN, LI) }\end{array}$ \\
\hline toutes et tous & 7 & $\begin{array}{l}\text { " Nous vous souhaitons à toutes et tous de bonnes vacances et de } \\
\text { bonnes fêtes de fin d'année ! (UGE, FB) }\end{array}$ \\
\hline
\end{tabular}

\subsection{Double flexion partielle}

Nous avons trouvé 21 exemples de la double flexion partielle (tableau 7). Les éléments lexicaux dédoublés de cette manière peuvent jouer de différents rôles dans la phrase. En ce qui concerne la façon d'orthographier de tels dédoublements, dans le corpus analysé nous avons trouvé deux caractères utilisés, notamment le point du bas (7 ex.) et le point médian (14 ex.).

Tableau 7

Double flexion partielle

\begin{tabular}{|c|c|c|}
\hline $\begin{array}{l}\text { Double flexion } \\
\text { partielle }\end{array}$ & $\begin{array}{l}\text { Nombre } \\
\text { d'emplois }\end{array}$ & Citation \\
\hline abonné·es & 1 & $\begin{array}{l}\text { "'L'Université Gustave Eiffel adapte ses formations pour } \\
\text { répondre aux nouveaux besoins de compétences' (édition } \\
\text { abonné es) via RGRA » (UGE, LI) }\end{array}$ \\
\hline chercheures & 2 & $\begin{array}{l}\text { "Retrouvez tous les articles de nos chercheur·es, enseignant·es } \\
\text { et doctorant·es sur notre page dédiée » (UGE, FB) }\end{array}$ \\
\hline doctorant.e.s & 1 & $\begin{array}{l}\text { «Doctorant.e.s de l'Université Paris Dauphine-PSL - (Univer- } \\
\text { sité PSL), présentez votre recherche en } 180 \text { secondes ! " (UPD- } \\
\text { PSL, LI) }\end{array}$ \\
\hline doctorantes & 2 & $\begin{array}{l}\text { "Retrouvez tous les articles de nos chercheur·es, enseignant·es } \\
\text { et doctorant·es sur notre page dédiée » (UGE, FB) }\end{array}$ \\
\hline engagées & 1 & $\begin{array}{l}\text { "Nos étudiant·es sont engagé·es dans le nouveau dispositif } \\
\text { \#Universanté en place dans notre université ! »(UGE, FB) }\end{array}$ \\
\hline enseignantes & 2 & $\begin{array}{l}\text { "Retrouvez tous les articles de nos chercheur'es, enseignant·es } \\
\text { et doctorant·es sur notre page dédiée » (UGE, FB) }\end{array}$ \\
\hline étudiant.es & 2 & $\begin{array}{l}\text { «Les agents et étudiant.es employés par l'Université vous rap- } \\
\text { pelleront et vous orienteront vers des aides internes ou externes } \\
\text { à l'Université » (UGE, FB) }\end{array}$ \\
\hline étudiant·es & 4 & $\begin{array}{l}\text { "Découvrez tous les aspects de ces études à travers une série de } \\
5 \text { vidéos de témoignages d'étudiant·es de l'école » (UGE, FB) }\end{array}$ \\
\hline
\end{tabular}




\begin{tabular}{|l|c|l|}
\hline $\begin{array}{l}\text { étudiant·es } \\
\text { ambassadeur-rices }\end{array}$ & 1 & «Retrouvez nos étudiant·es ambassadeur·rices » (UGE, FB) \\
\hline $\begin{array}{l}\text { étudiantes } \\
\text { engagées }\end{array}$ & 1 & $\begin{array}{l}\text { « Ces actions sont menées par le Crips Ile-de-Vœux, l'Agence } \\
\text { Régionale de Santé, notre Service de Santé Universitaire ainsi } \\
\text { que les étudiant·es engagé·es dans le dispositif Universanté » } \\
\text { (UGE, FB) }\end{array}$ \\
\hline intéressé.e & 3 & «Intéressé.e par un master en journalisme? ? (UPD-PSL, LI) \\
\hline seul.es & 1 & $\begin{array}{l}\text { "Vous n'êtes pas seul.es, l'Université vous accompagne. Prenez } \\
\text { soin de vous » (UGE, FB) }\end{array}$ \\
\hline
\end{tabular}

\subsection{Nom collectif}

Nous avons observé 36 noms collectifs dans le corpus (tableau 8). Certains de ces exemples ne sont pas strictement liés au champ lexical de l'université et de l'enseignement supérieur.

Les mots jeunesse et communauté présents dans le support textuel analysé sont deux exemples des noms collectifs appartenant à la sous-catégorie dite les noms de qualité. Il s'agit des cas où une caractéristique commence à désigner plusieurs porteurs. Mairie (de) Villetaneuse, Université, Université Gustave Eiffel, Université Sorbonne Paris Nord sont les mots collectifs qui appartiennent à la catégorie des noms d'institutions (N. Flaux, 1999 : 495 ; M. Lammert, M. Lecolle, $2014: 206)$.

Tableau 8

Nom collectif

\begin{tabular}{|c|c|c|}
\hline Nom collectif & $\begin{array}{l}\text { Nombre } \\
\text { d'emplois }\end{array}$ & Citation \\
\hline communauté & 1 & $\begin{array}{l}\text { "L'un des objectifs est de tripler, d'ici deux ans, les effectifs } \\
\text { d'\#alternants et de leur offrir un véritable parcours au sein de } \\
\text { Covéa, en créant une communauté [...]» (UPD-PSL, LI) }\end{array}$ \\
\hline équipe(s) & 5 & $\begin{array}{l}\text { "Avec John Kerry à la tête de la diplomatie \#climatique, } \\
\text { Washington va disposer d'une équipe particulièrement aguerrie } \\
\text { en la matière » (UPD-PSL, LI) }\end{array}$ \\
\hline jeunesse & 1 & « La jeunesse est un investissement d'avenir » (UPD-PSL, FB) \\
\hline $\begin{array}{l}\text { Mairie (de) } \\
\text { Villetaneuse }\end{array}$ & 3 & $\begin{array}{l}\text { "Christophe Fouqueré, président de l'Université Sorbonne Paris } \\
\text { Nord a été interviewé par la Mairie Villetaneuse, dans le cadre } \\
\text { de sa prise de fonctions [...]»(USPN, FB) }\end{array}$ \\
\hline personnel & 1 & $\begin{array}{l}\text { «Ce n'est pas seulement la gestion du personnel qui est ainsi } \\
\text { évitée }[\ldots] » \text { (UPD-PSL, LI) }\end{array}$ \\
\hline
\end{tabular}




\begin{tabular}{|l|c|l|}
\hline société & 6 & $\begin{array}{l}\text { «[...] l'Université Gustave Eiffel ainsi que sept autres établis- } \\
\text { sements publics, signent une charte d'ouverture à la \#société » } \\
\text { (UGE, FB) }\end{array}$ \\
\hline Université & 12 & $\begin{array}{l}\text { «L'Université va vous contacter pour une enquête d'insertion. » } \\
\text { (USPN, FB) }\end{array}$ \\
\hline $\begin{array}{l}\text { Université } \\
\text { Gustave Eiffel }\end{array}$ & 6 & $\begin{array}{l}\text { «L'Université Gustave Eiffel vous accompagne et vous propose } \\
\text { des solutions pour acquérir du matériel informatique. » (UGE, } \\
\text { FB) }\end{array}$ \\
\hline $\begin{array}{l}\text { Université } \\
\text { Sorbonne Paris } \\
\text { Nord }\end{array}$ & 1 & $\begin{array}{l}\text { «L'Université Sorbonne Paris Nord vous présente ses meilleurs } \\
\text { vœux 2021 ! (USPN, LI) }\end{array}$ \\
\hline
\end{tabular}

\subsection{Formule englobante}

La formule englobante a été observée 21 fois dans le corpus étudié. Communauté scientifique, figures de l'opposition et jeune équipe de recherche ne sont que quelques exemples de cette technique. Le tableau 9 rassemble plus d'expressions pareilles ainsi que les citations.

Tableau 9

Formule englobante

\begin{tabular}{|l|c|l|}
\hline \multicolumn{1}{|c|}{$\begin{array}{c}\text { Formule } \\
\text { englobante }\end{array}$} & $\begin{array}{c}\text { Nombre } \\
\text { d'emplois }\end{array}$ & \multicolumn{1}{c|}{ Citation } \\
\hline $\begin{array}{l}\text { communauté } \\
\text { dauphinoise }\end{array}$ & 1 & $\begin{array}{l}\text { «Une carrière qui rend fière la communauté dauphinoise » } \\
\text { (UPD-PSL, LI) }\end{array}$ \\
\hline $\begin{array}{l}\text { communauté } \\
\text { scientifique }\end{array}$ & 2 & $\begin{array}{l}\text { «[...] un investissement majeur auprès de la communauté scien- } \\
\text { tifique à l'échelle internationale. » (USPN, LI) }\end{array}$ \\
\hline $\begin{array}{l}\text { corps enseignant } \\
\text { équipes de } \\
\text { l'Université } \\
\text { Paris Dauphine }\end{array}$ & 2 & $\begin{array}{l}\text { « Notre corps enseignant vient de multiples horizons et déve- } \\
\text { loppe des méthodes d'apprentissage innovantes. » (UPD-PSL, FB) }\end{array}$ \\
\hline $\begin{array}{l}\text { équipes } \\
\text { managériales et } \\
\text { pédagogiques }\end{array}$ & 1 & $\begin{array}{l}\text { « Le président EM Mouhoud et les équipes de l'Université Paris } \\
\text { Dauphine - PSL vous présentent leurs meilleurs vœux pour la } \\
\text { nouvelle année » (UPD-PSL, FB) }\end{array}$ \\
\hline $\begin{array}{l}\text { équipes } \\
\text { pédagogiques }\end{array}$ & 4 & $\begin{array}{l}\text { et pédagogiques, ce grand commis de l'État est revenu sur sa } \\
\text { carrière, longue et riche d'expériences. » (UPD-PSL, FB) }\end{array}$ \\
\hline
\end{tabular}




\begin{tabular}{|l|c|l|}
\hline $\begin{array}{l}\text { figures de } \\
\text { l'opposition }\end{array}$ & 1 & $\begin{array}{l}\text { «Contestations à \#HongKong : 53 figures de l'opposition arrê- } \\
\text { tées ce mercredi » (UPD-PSL, LI) }\end{array}$ \\
\hline $\begin{array}{l}\text { jeunes équipes } \\
\text { de recherche }\end{array}$ & 2 & $\begin{array}{l}\text { « Appel à projets destiné aux jeunes équipes de recherche » } \\
\text { (USPN, LI) }\end{array}$ \\
\hline $\begin{array}{l}\text { nouvelle équipe } \\
\text { de recherche }\end{array}$ & 2 & $\begin{array}{l}\text { «Cet appel à projets vise à mettre en place et animer une nou- } \\
\text { velle équipe de recherche » (USPN, LI) }\end{array}$ \\
\hline $\begin{array}{l}\text { personnes } \\
\text { extérieures }\end{array}$ & 1 & $\begin{array}{l}\text { «Cours ouverts à tous les étudiants et personnels de l'université } \\
\text { mais également aux personnes extérieures. » (USPN, FB) }\end{array}$ \\
\hline $\begin{array}{l}\text { population } \\
\text { mondiale }\end{array}$ & 1 & $\begin{array}{l}\text { «[...] le fait d'exclure une grosse partie de la population mon- } \\
\text { diale aura des répercussions dramatiques » (UPD-PSL, LI) }\end{array}$ \\
\hline $\begin{array}{l}\text { responsables } \\
\text { d'institutions }\end{array}$ & 1 & $\begin{array}{l}\text { «De } 14 \mathrm{~h} 30 \text { à 18h30, suivez en direct les interventions de cher- } \\
\text { cheurs, grands témoins, et responsables d'institutions à l'occa- } \\
\text { sion de la première conférence annuelle de la Chaire \#Femmes et } \\
\text { \#Science » (UPD-PSL, LI) }\end{array}$ \\
\hline $\begin{array}{l}\text { toutes les } \\
\text { équipes de } \\
\text { l'université }\end{array}$ & 1 & $\begin{array}{l}\text { « Le président El Mouhoub Mouhoud et toutes les équipes de } \\
\text { l'université vous présentent leurs meilleurs vœux pour la nou- } \\
\text { velle année ! » (UPD-PSL, IG) }\end{array}$ \\
\hline
\end{tabular}

\subsection{Formule non-genrée}

Nous avons observé 33 formules non-genrées dans le corpus étudié. Elles sont les suivantes :

- association étudiante qui remplace association d'étudiants ou association des étudiants ;

- carte étudiante qui remplace carte d'étudiant;

- préoccupations citoyennes qui remplace préoccupation des citoyens;

- vie étudiante qui remplace vie d'étudiant.

L'exemple préoccupations citoyennes est le plus intéressant en raison de l'emploi du mot citoyen comme l'adjectif. Le TLFi qualifie ce lexème comme l'adjectif vieilli. La $9^{\mathrm{e}}$ édition du dictionnaire de l'Académie française ne donne aucune information sur l'emploi de ce mot en tant qu'adjectif ${ }^{22}$.

\footnotetext{
${ }^{22}$ https://www.cnrtl.fr/definition/academie9/citoyen (consulté le 12.02.2021).
} 
Formule non-genrée

\begin{tabular}{|l|c|l|}
\hline \multicolumn{1}{|c|}{$\begin{array}{c}\text { Formule } \\
\text { non-genrée }\end{array}$} & $\begin{array}{c}\text { Nombre } \\
\text { d'emplois }\end{array}$ & \multicolumn{1}{|c|}{ Citation } \\
\hline $\begin{array}{l}\text { association } \\
\text { étudiante }\end{array}$ & 15 & $\begin{array}{l}\text { « L'association étudiante 'Womansplaining' de l'université } \\
{[\ldots] \text { (... (USPN, FB) }}\end{array}$ \\
\hline carte étudiante & 1 & $\begin{array}{l}\text { « Trois masques lavables jusqu'à 60 fois vous seront distribués } \\
\text { (selon les stocks disponibles) sur présentation de votre carte étu- } \\
\text { diante et dans le respect des gestes barrières. » (UGE, FB) }\end{array}$ \\
\hline $\begin{array}{l}\text { préoccupations } \\
\text { citoyennes }\end{array}$ & 2 & $\begin{array}{l}\text { « Pour faire face aux préoccupations citoyennes [...] [ils] signent } \\
\text { une charte d'ouverture à la \#société. » (UGE, FB) }\end{array}$ \\
\hline vie étudiante & 15 & $\begin{array}{l}\text { « Élèves de Terminale, venez découvrir les formations, les cam- } \\
\text { pus, les modalités de candidature et la vie étudiante dauphinoise } \\
{[\ldots] \text { (UPD-PSL, LI) }}\end{array}$ \\
\hline
\end{tabular}

\subsection{Forme épicène}

Les mots épicènes trouvés dans les textes publiés par les universités sont les suivants : artiste(s) $\left(7^{23}\right)$, bénévoles (2), bibliothécaire universitaire (2), cétologue (2), économiste (15), effectifs (2), élèves de terminale (3), éthologue (2), fans (1), membre(s) (9), parents (2), personnels (5), porte-parole (1), responsable(s) (8), sociologue (1), spécialiste (3), stagiaires (1) et talents (1), témoins (1). Nous constatons que certains de ces mots ont remplacé leurs synonymes non-épicènes qui aussi bien auraient pu être utilisés à leur place. Nous démontrons les citations dans le tableau 11.

La dernière remarque est une mise en exergue de la réapparition du mot personnel déjà montré dans cet article comme l'exemple du nom collectif. La raison de cette résurgence est son utilisation au pluriel. Le TLFi donne la définition suivante du nom personnel : «Ensemble des personnes appartenant à une même profession ou à un même corps $»^{24}$. Dans la $9^{\mathrm{e}}$ édition du dictionnaire de l'Académie française, il est précisé :

Le nom Personnel est un nom collectif qui désigne toujours un ensemble d'individus. Il n'est donc acceptable au pluriel que si l'on veut désigner plusieurs catégories distinctes d'individus, par exemple les personnels civil et militaire des armées ${ }^{25}$.

Une fois qu'il est privé de sa valeur collective, le mot personnels est épicène et peut remplacer ses synonymes qui n'ont pas cette caractéristique.

${ }^{23}$ Le nombre d'emplois se trouve entre parenthèses.

${ }^{24} \mathrm{https} / /$ www.cnrtl.fr/definition/personnel/substantif (consulté le 12.02.2021).

${ }^{25}$ https://www.cnrtl.fr/definition/academie9/personnel/substantif (consulté le 12.02.2021). 
Forme épicène

Tableau 11

\begin{tabular}{|c|c|c|}
\hline Forme épicène & Citation & $\begin{array}{l}\text { Forme } \\
\text { non-épicène } \\
\text { remplacée } \\
\text { (exemples) }\end{array}$ \\
\hline artiste & $\begin{array}{l}\text { «L'artiste Rakajoo a réalisé deux fresques uniques pour } \\
\text { l’Université Gustave Eiffel [...]»(UGE, FB) }\end{array}$ & peintre \\
\hline effectifs & $\begin{array}{l}\text { "Afin de respecter les mesures sanitaires en vigueur, les } \\
\text { effectifs sont limités et certaines modalités sont mises en } \\
\text { places }^{26} \text { » (USPN, FB) }\end{array}$ & employés \\
\hline $\begin{array}{l}\text { élèves de } \\
\text { terminale }\end{array}$ & $\begin{array}{l}\text { «Élèves de Terminale, faites les bons choix sur Parcour- } \\
\text { sup ! » (UPD-PSL, FB) }\end{array}$ & lycéens \\
\hline personnels & $\begin{array}{l}\text { " \#HappyNewYear2021 \#Étudiants \#Personnels \#Ensei- } \\
\text { gnants \#Chercheurs \#BestWishes \#2021 » (UGEI, IG) }\end{array}$ & employés \\
\hline porte-parole & $\begin{array}{l}\text { «[...] étudiante en Master } 2 \text { Économie et Finance parcours } \\
\text { 'Énergie, Finance, Carbone' à l'Université Paris-Dauphine } \\
\text { - Université PSL, et porte-parole du mouvement Pour un } \\
\text { réveil écologique [...]» (UPD-PSL, LI) }\end{array}$ & représentant \\
\hline talents & $\begin{array}{l}\text { «En février, plus d'une centaine d'entreprises avaient pris } \\
\text { part au \#forum pour rencontrer leurs futurs talents à Dau- } \\
\text { phine - PSL » (UPD-PSL, LI) }\end{array}$ & employé \\
\hline
\end{tabular}

\subsection{Abréviation}

Nous avons sélectionné deux formes abréviatives ayant la valeur épicénisante. La première est $D r$ qui est une utilisation spécifique des lettres afin de réduire la graphie des mots docteur et docteure. Il s'agit d'une abréviation qui est commune pour les deux genres, et qui est apparue dans la phrase suivante :

Sous la direction du Dr Mathilde Touvier, Directrice de l'Équipe de Recherche en Épidémiologie Nutritionnelle (EREN) de l'Université Sorbonne Paris Nord, il a été récompensé pour ses travaux de thèse [...] (USPN, LI et FB).

La seconde abréviation observée dans le corpus est $V P$ qui a remplacé les mots vice-président et vice-présidente. Elle a été utilisée 24 fois dans les publications de l'Université Sorbonne Paris Nord. Voici un exemple qui illustre ses trois emplois :

${ }^{26}$ Nous présentons les citations sans intervenir sur l'orthographe même si elle n'est pas correcte. 
La distribution a lieu en présence de Faten Hidri, VP de la Région Île-de-France en charge de l'Enseignement supérieur et de la Recherche, de Farida Adlani, VP de la Région Île-de-France en charge de la Santé, Solidarité et de la Famille, Christophe Fouqueré, président de l'Université Sorbonne Paris Nord, Pascal Coupey, VP CFVU de l'Université Sorbonne Paris Nord (USPN, FB).

\subsection{Féminisation}

Les publications Internet des trois universités choisies pour l'étude nous ont permis d'observer 53 féminisations des noms intéressantes (tableau 12). Il sied de préciser que le support textuel de l'étude nous a donné en grande majorité des féminisations des noms entrant dans le champ lexical de l'université, p. ex. professeure, directrice de l'institut de recherche et vice-présidente.

La première chose à voir de plus près est la féminisation du substantif chercheur. Le TLFi et la $9^{\mathrm{e}}$ édition du dictionnaire de l'Académie française indiquent que le suffixe de la forme féminine est -euse $e^{27,28}$. Le dictionnaire Larousse précise que " [1]e féminin chercheuse est correct, mais dans l'usage courant, on emploie encore souvent le masculin pour désigner une femme $»^{29}$. La féminisation de ce mot avec le suffixe -euse a été observée trois fois dans le corpus de la recherche. Qui plus est, nous avons observé une féminisation de ce terme avec le suffixe -eure dans le composé lexical à trait d'union enseignante-chercheure. Cette féminisation a attiré notre attention parce que ni chercheure ni enseignante-chercheure ne sont pas référencés dans le TLFi, dans le dictionnaire Larousse, ni dans la $9^{e}$ édition du dictionnaire de l'Académie française.

Le nom suivant qui mérite sans doute une analyse plus détaillée est maîtresse de conférences. Le dictionnaire Larousse ne donne aucune variante féminine de ce lexème. Le TLFi indique le féminin maîtresse, mais il l'exclut du domaine de l'enseignement supérieur et de la recherche en indiquant que, dans ce contexte, le féminin et le masculin restent identiques ${ }^{30}$. Nous avons observé quatre utilisations de la forme masculine maître de conférences qui désignait une femme.

La dernière féminisation que nous voudrions commenter est directrice. Le dictionnaire Larousse et le TLFi confirment l'existence du féminin, mais dans le premier des dictionnaires nous trouvons l'information que « [...] dans les titres et les noms de fonctions, c'est en général la forme masculine qui est employée,

\footnotetext{
${ }^{27}$ https://www.cnrtl.fr/definition/chercheur/substantif (consulté le 12.02.2021).

${ }^{28} \mathrm{https}$ ://www.cnrtl.fr/definition/academie9/chercheur/substantif (consulté le 12.02.2021).

${ }^{29} \mathrm{https} / / /$ www.larousse.fr/dictionnaires/francais/chercheur/15135 (consulté le 12.02.2021).

${ }^{30} \mathrm{https} / / / \mathrm{www} . \mathrm{cnrtl}$.fr/definition/maitre (consulté le 12.02.2021).
} 
du moins en français de France $»^{31}$. Néanmoins, nous avons observé les noms de fonctions qui s'appuyaient sur la forme féminine :

- directrice de l'équipe de recherche,

- directrice de l'institut de recherche,

- directrice de l'UFR.

Nous n'avons pas observé d'utilisation de la forme masculine de ce mot dans le contexte où une femme était concernée.

Tableau 12

Féminisation

\begin{tabular}{|c|c|c|}
\hline Féminisation & $\begin{array}{l}\text { Nombre } \\
\text { d'emplois }\end{array}$ & Citation \\
\hline cheffe & 1 & $\begin{array}{l}\text { "Sous-Secrétaire générale de l’\#ONU pour l'Afrique, la Gui- } \\
\text { néenne Bintou Keita vient d'être nommée cheffe de la MO- } \\
\text { NUSCO }[\ldots] \text { (.. (UPD-PSL, LI) }\end{array}$ \\
\hline chercheuse & 3 & $\begin{array}{l}\text { "Intervention de l'économiste Anne-Laure Delatte, chercheuse } \\
\text { \#CNRS au sein du Laboratoire \#LED [...]» (UPD-PSL, LI). }\end{array}$ \\
\hline co-auteure & 2 & $\begin{array}{l}\text { "Lucile Desmoulins, maîtresse de conférences à l'Université } \\
\text { Gustave Eiffel, co-auteure de l'article [...]» (UGE, LI) }\end{array}$ \\
\hline $\begin{array}{l}\text { directrice de } \\
\text { l'équipe de } \\
\text { recherche }\end{array}$ & 2 & $\begin{array}{l}\text { "Sous la direction du Dr Mathilde Touvier, Directrice de } \\
\text { l'Équipe de Recherche en Épidémiologie Nutritionnelle (EREN) } \\
\text { de l'Université Sorbonne Paris Nord » (USPN, LI) }\end{array}$ \\
\hline $\begin{array}{l}\text { directrice de } \\
\text { l'institut de } \\
\text { recherche }\end{array}$ & 1 & $\begin{array}{l}\text { «La sociologue Dominique Méda, professeure de sociologie et } \\
\text { directrice de l'institut de recherche \#IRISSO à l'Université } \\
\text { Paris-Dauphine - PSL }[\ldots] \text { (UPD-PSL, LI) }\end{array}$ \\
\hline $\begin{array}{l}\text { directrice de } \\
\text { l'UFR }\end{array}$ & 11 & $\begin{array}{l}\text { «Une interview de Nathalie Coutinet, économiste de la santé, di- } \\
\text { rectrice de l'UFR SEG et professeure d'économie [...] » (USPN, } \\
\text { FB) }\end{array}$ \\
\hline $\begin{array}{l}\text { enseignante- } \\
\text { chercheure }\end{array}$ & 1 & $\begin{array}{l}\text { "[...] Janine Hobeika, enseignante-chercheure, Propedia Inc. » } \\
\text { (UPD-PSL, LI) }\end{array}$ \\
\hline $\begin{array}{l}\text { maîtresse de } \\
\text { conférences }\end{array}$ & 4 & $\begin{array}{l}\text { "Lucile Desmoulins, maîtresse de conférences à l'Université } \\
\text { Gustave Eiffel, co-auteure de l'article [...]»(UGE, LI) }\end{array}$ \\
\hline professeure & 23 & «Corine Pelluchon, professeure de \#philosophie [...]» (UGE, FB) \\
\hline $\begin{array}{l}\text { sous-secrétaire } \\
\text { générale }\end{array}$ & 1 & $\begin{array}{l}\text { "Sous-Secrétaire générale de l'\#ONU pour l'Afrique [...]" } \\
\text { (UPD-PSL, LI) }\end{array}$ \\
\hline vice-présidente & 4 & $\begin{array}{l}\text { «Félicitations à Anne Meddahi-Pellé, professeure des universités } \\
\text { et aujourd'hui vice-présidente du Conseil académique de l'uni- } \\
\text { versité Sorbonne Paris Nord }[. . .] » \text { (USPN, IG) }\end{array}$ \\
\hline
\end{tabular}

${ }^{31} \mathrm{https} / /$ www.larousse.fr/dictionnaires/francais/directeur/25775\#locution

(consulté le 12.02.2021). 


\section{En guise de conclusion}

Nous avons identifié 279 utilisations des techniques du FI dans les 356 publications examinées. Les trois universités françaises ont utilisé dans leurs médias sociaux huit pratiques du FI que nous listons rangées par ordre décroissant selon le nombre d'emplois :

- la forme épicène,

- la féminisation,

- le nom collectif,

- la formule non-genrée,

- l'abréviation,

- la double flexion totale,

- la double flexion partielle,

- la formule englobante.

Précisons que les trois dernières pratiques ont eu le même nombre d'emplois dans le corpus.

Le répertoire de pratiques démontre une remise en cause de la place du FI dans le mouvement de la déconstruction du paradigme du masculin, que ce soit dans la structure d'une langue ou d'une société. Ce qui est évidemment remarquable, c'est que malgré tous les efforts de l'Académie française pour la protection des principes de la langue, la prédominance du masculin est un élément qui n'échappe plus au regard attentif.

\section{Références citées}

Abbou, J. (2011). Double gender marking in French: a linguistic practice of antisexism. Current Issues in Language Planning, 12(1), 55-75.

Abbou, J., et al. (2018). Qui a peur de l'écriture inclusive ? Entre délire eschatologique et peur d'émasculation : Entretien. Semen. Revue de sémio-linguistique des textes et discours, 44, 133-150.

Académie française (1995). Statuts et règlements. Avec une note liminaire de Maurice Druon. https://www.academie-francaise.fr/sites/academie-francaise.fr/files/statuts_af_0.pdf(consulté le 14.03.2021).

Académie française (2014). La féminisation des noms de métiers, fonctions, grades ou titres. Mise au point de l'Académie française. http://www.academie-francaise.fr/sites/ academie-francaise.fr/files/feminisation_2014.pdf (consulté le 15.03.2021).

Académie française (2017). Déclaration de l'Académie française sur l'écriture dite « inclusive » adoptée à l'unanimité de ses membres dans la séance du jeudi 26 octobre 
2017. http://www.academiefrancaise.fr/actualites/declaration-de-lacademie-francaisesur-lecriture-dite-inclusive (consulté le 14.03.2021).

Académie française (2019). La féminisation des noms de métiers et de fonctions. https:// www.academie-francaise.fr/sites/academie-francaise.fr/files/rapport_feminisation noms_de_metier_et_de_fonction.pdf (consulté le 14.03.2021).

Académie française (2021). Lettre ouverte sur l'écriture inclusive. https://www.academiefrancaise.fr/actualites/lettre-ouverte-sur-lecriture-inclusive (consulté le 12.07.2021).

Aeschlimann, R. J. (2017). Un genre de nouvelle discrimination chromatique. GLAD!, 3, $529-566$.

Alpheratz, (2019). Français inclusif : du discours à la langue ? Le discours et la langue, $11(1), 53-74$.

Amade, J. S. (2018). L'écriture et les signes inclusif·ve·s, avec ou sans ? Activité pédagogique de Français sur Objectifs Spécifiques. Caligrama: Revista de Estudos Românicos, 23(2), 41-74.

Bereni, L., et al. (2012). Introduction aux études sur le genre. Bruxelles, De Boeck.

Bolter, F. (2019). « Le masculin l'emporte » : évolution des stratégies linguistiques dans les associations LGBT+ en France. H-France Salon, 11(14), 1-12.

Chatard, A., Guimond, S., \& Martinot, D. (2005). Impact de la féminisation lexicale des professions sur l'auto-efficacité des élèves : Une remise en cause de l'universalisme masculin ? L'Année Psychologique, 105(2), 249-72.

Dawes, E. (2003). La féminisation des titres et fonctions dans la Francophonie : de la morphologie à l'idéologie. Ethnologies, 25(2), 195-213.

Elmiger, D. (2008). Abréger les femmes pour mieux les nommer : féminisation de la langue et techniques abréviatives. Sêméion, 6, 119-125.

Elmiger, D. (2014). Cachez ces doublons que je ne saurais voir : les doubles formes féminine et masculine dans le langage administratif suisse. Cahiers de linguistique, 40(1), $155-170$.

Elmiger, D. (2017). Binarité du genre grammatical — binarité des écritures ? Mots. Les langages du politique, 1, 37-52.

Elmiger, D. (2018). Les genres récrits : chronique $n^{\circ} 3$. Au-delà de la binarité : le trouble entre les genres. GLAD!, 4, 908-927.

Elmiger, D. (2019). Les genres récrits : chronique $n^{\circ}$ 6. Qu'est-ce que le genre - et à qui appartient son interprétation ? GLAD!, 7, 615-637.

Elmiger, D. (2020). Les genres récrits : chronique $n^{\circ} 7$. Le féminin générique ou : une généricité peut en cacher une autre. GLAD!, 9, 696-726.

Fairon, C., \& Simon, A.-C. (2018). D'après l'œuvre de Grevisse. Le petit Bon usage de la langue française. Grammaire. Bruxelles, De Boeck Supérie.

Flaux, N. (1999). À propos des noms collectifs. Revue de linguistique romane, 63, 471502.

Gygax, P., Gabriel, U., \& Zufferey, S. (2019). Le masculin et ses multiples sens : Un problème pour notre cerveau... et notre société. Savoirs en Prisme, 10, 57-72.

Lammert, M. (2017). «Une sorte de nom collectif» : lecture catégorielle et lecture approximative. Syntaxe et sémantique, 18(1), 101-116.

Lammert, M., \& Lecolle, M. (2014). Les noms collectifs en français, une vue d'ensemble. Cahiers de Lexicologie, 105(2), 203-222. 
Michel, L. (2016). Penser la primauté du masculin - sémantique du genre grammatical, perspectives synchroniques et diachroniques. SHS Web of Conferences, 27, 1-23.

Omer, D. (2020). La fin du masculin générique ? Expériences et débats autour de l'écriture inclusive. Romanica, 31, 181-202.

Thireau, I. (2014). Les mobilisations collectives pour la défense de l'environnement et de la sécurité alimentaire. Informations sociales, 185(5), 112-118.

Viennot, É. (2020). Langage égalitaire : vers une rationalisation des procédés et des approches. Cahiers d'histoire. Revue d'histoire critique, 146, 149-160.

\section{Sitographie}

http://www.slate.fr/story/153492/manifeste-professeurs-professeures-enseignerons-plusmasculin-emporte-sur-le-feminin (consulté le 14.03.2021).

https://www.assemblee-nationale.fr/dyn/15/rapports/ega/115b1095_rapport-information (consulté le 13.03.2021)

https://www.cnrtl.fr/definition/abr\%C3\%A9viations (consulté le 16.07.2021).

https://www.cnrtl.fr/definition/academie9/chercheur/substantif (consulté le 12.02.2021).

https://www.cnrtl.fr/definition/academie9/citoyen (consulté le 12.02.2021).

https://www.cnrtl.fr/definition/academie9/personnel/substantif (consulté le 12.02.2021). https://www.cnrtl.fr/definition/chercheur/substantif (consulté le 12.02.2021).

https://www.cnrtl.fr/definition/personnel/substantif (consulté le 12.02.2021).

https://www.larousse.fr/dictionnaires/francais/chercheur/15135 (consulté le 12.02.2021).

https://www.larousse.fr/dictionnaires/francais/directeur/25775\#locution (consulté le

12.02.2021).

https://www.lefigaro.fr/actualite-france/2017/09/22/01016-20170922ARTFIG00300-un-

manuel-scolaire-ecrit-a-la-sauce-feministe.php (consulté le 14.03.2021).

https://www.larousse.fr/dictionnaires/francais/acronyme/858 (consulté le 10.04.2021).

https://www.cnrtl.fr/definition/troncation (consulté le 10.04.2021).

https://www.cnrtl.fr/definition/citoyen (consulté le 12.02.2021).

https://www.cnrtl.fr/definition/maitre (consulté le 12.02.2021).

https://www.delta-atled.org/ (consulté le 23.02.2021).

https://blogterrain.hypotheses.org/11453 (consulté le 13.03.2021).

https://www.cnrtl.fr/definition/sigle (consulté le 10.04.2021).

\section{Annexe}

Le corpus de la recherche a été publié sur Dropbox et est disponible sur le lien suivant : https://www.dropbox.com/s/9otvj0yz0sc4vzj/S\%E2\%80\%99attaquer\%20 $\% \mathrm{C} 3 \% \mathrm{~A} 0 \% 201 \mathrm{a} \% 20$ supr $\% \mathrm{C} 3 \% \mathrm{~A} 9$ matie $\% 20 \mathrm{du} \% 20$ masculin $\% 20$ sur $\% 201 \mathrm{e} \% 20$ f\%C3\%A 9minin $\% 20-\% 201$ e $\% 20$ fran $\%$ C3\%A7ais $\% 20$ inclusif $\% 20$ dans $\% 201$ es $\% 20$ publications $\% 20 \mathrm{des} \% 20$ universit $\% \mathrm{C} 3 \% \mathrm{~A} 9 \mathrm{~s} \% 20$ fran $\% \mathrm{C} 3 \%$ A 7 aises $\% 20 \mathrm{dans} \% 20$ les $\% 20$ r $\%$ C3\%A9seaux $\% 20$ sociaux $\% 20$-\%20CORPUS $\% 20$ DE $\% 20$ LA $\% 20$ RECHERCHE.pdf?dl $=0$

Il existe également une version raccourcie du lien : https://tiny.pl/9rcdj. 